\title{
Der internationale Terrorismus als Herausforderung des deutschen Strafrechts - Erwägungen zu einer Gratwanderung
}

\author{
Milan Kuhli*
}

\begin{abstract}
The phenomenon of terrorism makes people fear about the internal security in Germany - a fear which results in the demand for state reactions. This article asks to what extent criminal law is a proper instrument with which to come to terms with the phenomenon of terrorism. Unlike "ordinary" criminals, terrorists embrace a system of absolute beliefs and convictions. But in democratic cultures, such absolute beliefs and convictions are subject to the outcomes of democratic procedures, and thus the terrorist necessarily stands outside such a culture. Criminal law in a rule of law state fundamentally depends on communicating with criminals - sending a message of deterrence, for example, or of condemnation, or serving any of a number of other expressive functions. And the question with terrorists whose views are so absolute as to be unalterable is whether such communication is possible at all. These are practical limits to the use of criminal law as an instrument with which to deal with terrorism, but there are also normative limits, which are discussed in this article as well.
\end{abstract}

Keywords: Strafrecht, Terrorismus, Rechtsstaat, Sicherheit

Criminal law, terrorism, rule of law, security

\section{Einleitung}

$\mathrm{F}$ ür das Verhältnis des Rechtsstaats zum Terrorismus scheint es seit Jahrhunderten eine passende Losung zu geben: „Keine Freiheit für die Feinde der Freiheit!“. Dieser Kampfspruch der wehrhaften Demokratie vermag in seiner Kürze und seiner doppelten Belegung des Begriffs der Freiheit gleichermaßen zu faszinieren und zu überzeugen. Nicht zuletzt deshalb wird er auch heute noch in der politischen Debatte verwendet, so in der jüngeren Vergangenheit im Rahmen der Diskussion um ein Verbot rechtsradikaler Parteien. ${ }^{1}$ Nicht vergessen werden darf jedoch auf der anderen Seite, dass die genannte Losung in erheblichem Maße wertausfüllungsbedürftig ist. Die Verwendung dieser Parole verlangt einen Konsens darüber, was im Einzelfall unter den Begriffen Freiheit, Feind und nochmals Freiheit verstanden wird. Dass die Auffassungen hierzu erheblich divergieren können, zeigt bereits die Entstehungsgeschichte dieser Forderung. Ihre Urheberschaft verdankt sie dem französischen Revolutionär Louis Jean Saint-Just, einem der fanatischsten Köpfe der Französischen Revolution, den man wegen seiner Rolle im Wohlfahrtsausschuss auch den „Erzengel des Terrors“ oder den „Erzengel des Todes“ nannte. ${ }^{2}$ Die Grenzerfahrung der Aufklärung mit dem Totalitarismus in der Französischen Revolution mag auch dem modernen Rechtsstaat eine Warnung davor sein, welche Gratwanderung der Kampf gegen den Terrorismus mit sich bringt. Die Demokratie und der Rechtsstaat

Dr. jur. Milan Kuhli, M.A., Assessor jur, hat Rechtswissenschaft, Mittlere und Neuere Geschichte sowie Politologie studiert. Zurzeit arbeitet er als Wissenschaftlicher Mitarbeiter bei Professor Dr. jur. Klaus Günther im ExzellenzCluster „Die Herausbildung normativer Ordnungen“ an der Johann Wolfgang Goethe-Universität Frankfurt am Main.

1 Vgl. Claus Christian Malzahn, Keine Freiheit für die Feinde der Freiheit, in: http://www.spiegel.de/politik/debatte/0,1518,438225,00.html [11. Januar 2010].

2 Stefan Dietrich, Wehrhafte Demokratie, in: Frankfurter Allgemeine Zeitung vom 30. Mai 2008, S. 1; vgl. Norman Hampson, Saint-Just. Erzengel des Todes, Göttingen 1992. Vgl. überdies zu Saint-Just: Jörg Monar, Saint-Just. Sohn, Denker und Protagonist der Revolution, Bonn 1993; Bernard Vinot, Saint-Just, Stuttgart 1989. geraten dann in Gefahr, wenn durch Terroranschläge der Boden bereitet wird für autoritäre Gegenmaßnahmen, die die offene Gesellschaft in Frage stellen und damit ihrerseits zu einer Gefahr für die innere Sicherheit werden. ${ }^{3}$ Vor dem Hintergrund dieses Spannungsverhältnisses zwischen terroristischer Bedrohung und dem Risiko staatlicher Überreaktion stellt sich die Frage, was das deutsche Strafrecht zur Bekämpfung von Terrorismus beitragen kann - und beitragen darf.

\section{Zum Begriff des Terrorismus}

Die Debatte über die Reaktionsmöglichkeiten auf Terrorismus wird dadurch erschwert, dass es an einer allgemein anerkannten Definition dessen fehlt, was man unter Terrorismus zu verstehen hat. Zum einen existiert ein ganzes Konglomerat potenziell terroristischer Handlungen, das in seiner Vielfalt vom heimlich agierenden Bombenleger einer Terrorzelle bis zum Staatsterror der Gestapo reicht. ${ }^{4}$ Man findet nicht nur Unterschiede in den objektiven Ausdrucksformen, sondern auch in den subjektiven Gründen und Absichten der Täter. ${ }^{5}$ Zum anderen ist der Terrorismusbegriff in erheblicher Weise politisch besetzt und dementsprechend teilweise äußerst umstritten. Dies beginnt bereits damit, dass das Wort Terrorismus seine negative Konnotation erst im frühen 20. Jahrhundert erhielt, als der Sozialdemokrat Karl Kautsky die russischen Bolschewiken als „Terroristen“ bezeichnete, während la terreur für den bereits erwähnten Saint-Just eine positiv besetzte Methode zur Vernichtung der

3 Cornelius Prittwitz, Krieg als Strafe - Strafrecht als Krieg. Wird nach dem „11. September" nichts mehr sein, wie es war?, in: Ders./Michael Baurmann/Klaus Günther (Hrsg.), Festschrift für Klaus Lüderssen, Baden-Baden 2002, S. 499, 501.

4 Vgl. Eckart Klein, Die Herausforderung durch den internationalen Terrorismus - hört hier das Völkerrecht auf?, in: Josef Isensee (Hrsg.), Der Terror, der Staat und das Recht, Berlin 2004, S. 9, 10 f.

5 Vgl. Kai Hirschmann, Internationaler Terrorismus gestern und heute: Entwicklungen, Ausrichtung, Ziele, in: Hans Frank/Kai Hirschmann (Hrsg.), Die weltweite Gefahr. Terrorismus als internationale Herausforderung, Berlin 2002, S. 27, 35; Eckart Klein, a.a.O., S. 9, 11. 
Revolutionsgegner war. ${ }^{6}$ Als weiteres Beispiel für die politische Besetztheit des Begriffes Terrorismus sei hier auch der Definitionsstreit genannt, der im Zusammenhang mit dem seit dem 11. September 2001 aufkeimenden Vorschlag entbrannte, das Statut des Internationalen Strafgerichtshofs um das Delikt des Terrorismus zu erweitern. Dieser politische Streit dreht sich unter anderem um die Frage, ob auch Handlungen von Staaten als Terrorismus eingestuft werden sollen. Darüber hinaus kann jenseits dieses Randbereichs der Begrifflichkeit im Einzelfall immer umstritten sein, ob eine Aktion die eines „Freiheitskämpfers“ oder die eines „Terroristen“ darstellt. ${ }^{7}$ Eine solche semantische Grenzziehung, die häufig von der politischen Perspektive des Betrachters abhängt, wird noch dadurch erschwert, wenn man zusätzlich Begriffe wie „Aufständische“ oder „Separatisten“ ins Spiel bringt. Die Selbsteinschätzung der Akteure hilft hier wenig weiter, wie das historische Beispiel der Roten Armee Fraktion (RAF) zeigt: Wie diese, würde sich auch heute kaum ein Terrorist als ein solcher bezeichnen. ${ }^{8}$

Will man einem Widerstandsrecht des Einzelnen gegen ein ihn belastendes Unrechtsregime nicht von vornherein die Legitimation versagen - so schreibt beispielsweise John Locke ausgehend von der Idee des Gesellschaftsvertrages: „ungerechter und ungesetzlicher Gewalt darf Gewalt entgegengesetzt werden “9 -, so wird tatsächlich eine Abgrenzung des legitimen Freiheitskampfes gegenüber illegitimem Terror nötig. Kriterien dieser Abgrenzung können nur der „Gegner“ und die Wahl der Mittel sein. Die Annahme eines Freiheitskampfes gegen einen demokratischen Staat erscheint von vornherein abwegig. Auch muss eine absichtlich herbeigeführte Verletzung von Zivilisten die Bejahung eines legitimen Freiheitskampfes ausschließen. ${ }^{10}$ So klar eine Abgrenzung an diesen extremen Enden der Skala vorgenommen werden kann, so augenscheinlich ist auf der anderen Seite zugleich, dass es in der Großzahl der Fälle unterschiedliche politische Interpretationsmodelle geben kann. Gleichwohl existieren auch auf völkerrechtlicher Ebene immer wieder Versuche einer Skizzierung - der Verfasser vermeidet den Begriff der Definition - dessen, was Terrorismus ist. Beispielsweise umschreibt Art. 2 Abs. 1 lit. b des Internationalen Übereinkommens zur Bekämpfung der Finanzierung des Terrorismus vom 9. Dezember 1999 terroristische Handlungen unter anderem als solche, „die den Tod oder eine schwere Körperverletzung einer Zivilperson oder einer anderen Person, die bei einem bewaffneten Konflikt nicht aktiv an den Feindseligkeiten teilnimmt, herbeiführen soll, wenn diese Handlung aufgrund ihres Wesens oder der Umstände darauf abzielt, die Bevölkerung einzuschüchtern oder eine Regierung oder internationale Organisation zu einem Tun oder Unterlassen zu nötigen". ${ }^{11}$

6 Henner Hess, Discussing Terrorism, in: Cornelius Prittwitz/Michael Baurmann/Klaus Günther (Hrsg.), Festschrift für Klaus Lüderssen, Baden-Baden 2002, S. 489.

7 Gunther Hauser, Europa und der Kampf gegen den Terrorismus, in: Ders./Gerald Brettner-Messler (Hrsg.), Sicherheit und Recht zu Beginn des 21. Jahrhunderts, Wien 2007, S. 11, 16.

8 Heinz Gärtner, Robin Hood vs. Osama bin Laden: Rebellen, Freiheitskämpfer, Terroristen, in: Gunther Hauser/Gerald Brettner-Messler (Hrsg.), Sicherheit und Recht zu Beginn des 21. Jahrhunderts, Wien 2007, S. 69, 71f.

9 John Locke, Zwei Abhandlungen über die Regierung. Übersetzt v. Hans Jörn Hoffmann. Hrsg. u. eingeleitet v. Walter Euchner, Frankfurt a. M. 2004, Buch II, 18. Kapitel, § 204 (S. 328).

10 Vgl. Heinz Gärtner, a.a.O., S. 69, 76.

11 Unverbindliche deutsche Übersetzung, zitiert nach: BGBl. II (2003), S. 1923, 1927.
Wagt man einen Blick auf wissenschaftliche Begriffsbildung jenseits politischer Debatten, so lässt sich konstatieren, dass sich hinsichtlich wesentlicher Elemente dessen, was Terrorismus ausmacht, Einigkeit abzeichnet, ${ }^{12}$ wenn auch zuzugeben ist, dass unter den Begriff des Terrorismus recht unterschiedliche Verhaltensstrategien gefasst werden. ${ }^{13}$ Nach dieser Begriffsbildung ist terroristischen Akten gemein, dass sie sich als besondere Form der Gewaltanwendung mit im weitesten Sinne politischer Zielsetzung präsentieren, ${ }^{14}$ die über den konkreten Erfolg der jeweiligen terroristischen Handlung hinausgeht. Die Fernziele sollen über die psychischen Effekte physischer Gewalt erreicht werden. ${ }^{15}$ Terrorismus ist damit eine Strategie der Angsterzeugung. ${ }^{16}$ Das terroristische Mittel ist nicht die Zerstörung, sondern die bewusste Hervorrufung von Furcht und Schrecken. ${ }^{17}$ Was der Terrorist vor allem bezweckt, ist eine psychologische Schwächung seines Gegners. ${ }^{18}$ Er verfolgt mit seinen Aktionen die Absicht, in der terrorisierten Gesellschaft Angst hervorzurufen und das von ihm aus feindliche System zu destabilisieren. ${ }^{19}$ Darüber hinaus kann das angestrebte Ziel auch in der Beeinflussung konkreter politischer Entscheidungsprozesse bestehen. ${ }^{20}$ Terroristen führen häufig keine offene Auseinandersetzung, sondern agieren verdeckt. ${ }^{21}$ Der Schrecken - der Terror - wird gerade dadurch verbreitet, dass es vom Zufall abzuhängen scheint, wer Opfer derartiger Taten wird. Terroristische Gewaltanwendung stellt für den Akteur eine taugliche Methode dar, seine auf demokratischem Wege regelmäßig nicht durchsetzbaren Meinungen zu vermitteln. ${ }^{22}$ Der Terrorist als Idealtypus handelt dabei selbstlos, da es ihm nicht um materiellen Gewinn geht. Das Streben nach Macht dient ihm nicht dazu, diese nach Erlangung zu genießen, sondern dazu, seine Ideale umzusetzen. ${ }^{23}$ Doch bleibt dieser Idealtypus oft unerfüllt. Terrorismus ist für die Akteure in gewisser Weise auch Selbstzweck, indem er diesen Personen ein Maß an Macht gewährt, das zu ihrer eigentlichen Bedeutung völlig disproportional ist. ${ }^{24}$ So geriert sich das Terrornetzwerk Al Kaida mehr und mehr als globales Label, das für bestimmte Personengruppen bereits per se eine bestimmte Strahlkraft ausübt. Da es einem Franchise-System vergleichbar aufgebaut ist, ${ }^{25}$ ist es für den Einzelnen zudem relativ einfach, an diesem Label zu partizipieren.

Anstelle der phänomenologischen Begriffsbildung lässt sich die Erscheinung des Terrorismus auch staatstheoretisch deuten. Eine solche Begriffsbildung des Terrorismus ist in Anleh-

12 Vgl. Eckart Klein, a.a.O., S. 9, 11

13 Herfried Münkler, Terrorismus und demokratischer Rechtsstaat, in: WestEnd. Neue Zeitschrift für Sozialforschung 2/2006, S. 86.

14 Vgl. Eckart Klein, a.a.O., S. 9, 11.

15 Vgl. Herfried Münkler, WestEnd. Neue Zeitschrift für Sozialforschung 2/2006, S. 86.

16 Vgl. Benjamin R.. Barber, Ein Krieg ,jeder gegen jeden“: Terror und die Politik der Angst, in: APuZ 18/2002, S. 7.

17 Herfried Münkler, WestEnd. Neue Zeitschrift für Sozialforschung 2/2006, S. 86.

18 Gunther Hauser, a.a.O., S. 11.

19 Josef Isensee, Nachwort: Der Terror und der Staat, dem das Leben lieb ist, in: Ders. (Hrsg.), Der Terror, der Staat und das Recht, S. 83, 83f.

20 Gunther Hauser, a.a.O., S. 11.

21 Bernd Grzeszick, Staat und Terrorismus. Eine staatstheoretische Überlegung in praktischer Absicht, in: Josef Isensee (Hrsg.), Der Terror, der Staat und das Recht, S. 55, 63

22 Eckart Klein, a.a.O., S. 9f., 37.

23 Josef Isensee, a.a.O., S. 83, 85

24 Eckart Klein, a.a.O., S. 9, 37.

25 Gunther Hauser, a.a.O., S. 11, 13f. 
nung an Bernd Grzeszick ${ }^{26}$ in einem Kontrast zur modernen Staatlichkeit zu sehen. Der demokratische Staat kennt nicht den unbedingten Vorrang einer einzelnen Meinung, einer einzelnen materialen Wahrheit. Demokratie bedeutet Konfliktschlichtung mit Worten und nicht mit Gewalt. ${ }^{27}$ Demgegenüber verabsolutiert ein Terrorist sein politisches Ziel vor allen anderen politischen Ansichten. ${ }^{28}$ Dieser von ihm propagierte unbedingte Vorrang geht für ihn so weit, dass ihm für die Verwirklichung dieses Zieles nahezu jedes Mittel recht ist, unter Umständen auch das der Gewaltanwendung. Nach dem oben Gesagten erscheint die Gewaltanwendung für den Terroristen sogar als das einzige Mittel, da er seine Ansichten regelmäßig nicht mit demokratischen Mitteln durchzusetzen vermag. ${ }^{29}$ Indem der Terrorist sein politisches Ziel verabsolutiert und hierfür sogar Gewalt einzusetzen bereit ist, beansprucht er für sein Ziel nicht nur den Vorrang vor anderen politischen Anschauungen, sondern auch vor dem staatlichen Gewaltmonopol. Unter diesem staatstheoretischen Blickwinkel erweist sich Terrorismus als Angriff auf die moderne Staatlichkeit selbst, gewissermaßen als Kampf gegen die Kultur. ${ }^{30}$

\section{Der internationale Terrorismus als Heraus- forderung des deutschen Strafrechts}

Es liegt auf der Hand, dass es sich bei terroristischen Aktivitäten regelmäßig um Kriminalität handelt, so dass die Anknüpfung für strafrechtliche Sanktionen ohne Weiteres gegeben zu sein scheint. Gleichwohl - so die Ausgangsthese - stellt ein Terrorist für die deutsche Strafrechtsordnung ein „ungewohntes Subjekt" dar. Strafrecht ist nämlich für sämtliche der ihm zugeschriebenen Wirkungen auf Kommunikation angewiesen: Es diskriminiert ${ }^{31}$ und ist nach dem hergebrachten Konzept strafrechtlicher Prävention dazu berufen, durch die Sanktionierung auf die Psyche von Straftätern einzuwirken, damit diese von zukünftigen Straftatverwirklichungen abgehalten werden. ${ }^{32} \mathrm{Ne}-$ ben dieser Zielbestimmung soll strafrechtliche Prävention auch der Abschreckung und Normstabilisierung der Allgemeinheit dienen und damit auf unterschiedlichen Wegen die Gefahr der Verbrechensbegehung minimieren. ${ }^{33}$ Die Herausforderung des Terrorismus für das Strafrecht besteht darin, dass Terroristen aus kriminologischer Perspektive ihr Tun für gerechtfertigt halten. Zwar besitzen sie regelmäßig im strafrechtlichen Sinne volles Unrechtsbewusstsein, doch ist häufig das moralische Unrechtsbewusstsein nicht vorhanden. Terroristen halten

26 Vgl. zum Folgenden: Bernd Grzeszick, a.a.O., S. 55, 59ff.

27 Benjamin R. Barber APuZ 18/2002, S. 7.

28 Vgl. hierzu den Essay von Hans Magnus Enzensberger mit dem Titel „Die Träumer des Absoluten“, in: Ders., Politische Kolportagen, Frankfurt a. M. 1966, S. $169 \mathrm{ff}$.

29 Vgl. Eckart Klein, a.a.O., S. 9, 37.

30 Insoweit in Abweichung zu Grzeszick, der in Anlehnung an die deutsche Übersetzung von Samuel Huntington vom „Kampf der Kulturen“ spricht (Bernd Grzeszick, a.a.O., S. 55, 68).

31 Gerd Roellecke, Der Rechtsstaat im Kampf gegen den Terror, in: JZ 2006, S. 265 , 266.

32 Bettina Weißer, „Kampf gegen den Terrorismus“- Prävention durch Strafrecht, in: JZ 2008, S. 388, 393.

33 Winfried Hassemer, Gefahrenabwehr durch Strafrecht?, in: WestEnd. Neue Zeitschrift für Sozialforschung 2/2006, S. 75, 82. - Kritisch hierzu: Klaus Günther Der strafrechtliche Schuldbegriff als Gegenstand einer Politik der Erinnerung in der Demokratie, in: Gary Smith/Avishai Margalit (Hrsg.), Amnestie oder Die Politik der Erinnerung in der Demokratie, Frankfurt a. M. 1997, S. 48, 53 . ihren Verstoß gegen die Rechtsordnung, den sie als solchen erkennen, im Sinne ihres Kampfes für Gerechtigkeit, Freiheit oder ihre religiösen Überzeugungen subjektiv für richtig. ${ }^{34}$ Die von Terroristen verfochtene Ideologie wird in ihren Augen von einem absoluten und uneingeschränkten Wahrheitsanspruch getragen, was ihnen eine starke subjektive Rechtfertigungsmöglichkeit für ihre kriminellen Taten verschafft. Die - gegenständlich unbegrenzte - Ideologie von Terroristen arbeitet damit perfekt als Technik der Neutralisation, also als Mittel der Verleugnung der Opfer und Verdrängung des Unrechts. Auch nehmen viele terroristische Gruppen für sich in Anspruch, einen „Krieg“ zu führen, womit sie in ihrer subjektiven Sicht eine Klassifizierung ihres Verhaltens als „kriminell“ bestreiten können und überdies eine zusätzliche subjektive Rechtfertigung für den Umstand erhalten, dass auch unbeteiligte Personen zu Schaden kommen. ${ }^{35}$ Die demokratische Gesellschaft vermag einen Terroristen, der seine aus seiner Sicht für richtig gehaltene Ideologie verabsolutiert, nicht zu verstehen. Ist hier noch Raum für eine Kommunikation?

Das zuerst genannte Phänomen - die Neutralisierungstechnik des Terroristen - hindert für sich genommen die deutsche Strafrechtsordnung nicht, den Terroristen für seine Tat zu bestrafen. Ausreichend ist das Vorliegen eines strafrechtlichen Unrechtsbewusstseins. Jedoch stellt sich die Frage, ob im Bereich des Terrorismus von einem Funktionieren strafrechtlicher Prävention auszugehen ist. ${ }^{36}$ Ein völlig selbstlos agierender Terrorist, der bereit ist, für seine Ziele zu sterben, ist kein utilitaristisch denkender Mensch, der sich durch das Risiko einer strafrechtlichen Sanktion beeinflussen ließe. Gerd Roellecke mag deshalb darin Recht zu geben sein, dass der Rechtsstaat mit Terroristen grundsätzlich nicht kommunizieren kann, und zwar nicht nur im Falle von Selbstmordattentätern, bei denen die Unmöglichkeit der Kommunikation besonders offenkundig wird ${ }^{37}$ : Im Gegensatz zu „gewöhnlichen“ Verbrechern, die überleben wollen und damit also mindestens diesen Aspekt der Zugehörigkeit zur Gemeinschaft bewahren möchten, trifft diese Eigenschaft auf Selbstmordattentäter nicht zu, womit infolge des Fehlens individueller Selbsterhaltungsstrategien dem Kontraktualismus der Boden entzogen zu werden scheint. ${ }^{38}$ Bleibt bei diesen - oder bei nichtsuizidalen Terroristen - noch Raum für Prävention durch Strafrecht?

Gerade gegenüber der Personengruppe terroristischer Selbstmordattentäter, die fest entschlossen sind, ihr Leben zu opfern, werden seit Längerem auch Alternativen staatlicher Präventionsinstrumente diskutiert. ${ }^{39}$ Als ein Mittel, das auf das besondere Wertebewusstsein von Selbstmordattentätern abstellen soll, lässt beispielsweise der Staat Israel Häuser ihrer

34 Gerd Roellecke JZ 2006, S. 265, 268.

35 Hans-Heiner Kühne, Unzeitgemäße Betrachtungen zum Problem des Terrorismus, in: Thomas Feltes/Christian Pfeiffer/Gernot Steinhilper (Hrsg), Kriminalpolitik und ihre wissenschaftlichen Grundlagen. Festschrift für Hans-Dieter Schwind, Heidelberg 2006, S. 103, $103 \mathrm{f}$.

36 Vgl. Bettina Weißer JZ 2008, S. 388, 393.

37 Gerd Roellecke JZ 2006, S. 265, 268.

38 Regina Harzer, Staaten im globalen Naturzustand? Eine Stellungnahme zu den Attentaten in Amerika am 11. September 2001, in: Cornelius Prittwitz/Michael Baurmann/Klaus Günther (Hrsg.), Festschrift für Klaus Lüderssen, BadenBaden 2002, S. 481, 484.

39 Bernd Grzeszick, a.a.O., S. 55, 71 - Vgl. zu den israelischen Kollektivsanktionen gegen Familienangehörige von Selbstmordattentätern: Alan M. Dershowitz, Why terrorism works. Understanding the threat, responding to the challenge, New Haven/London 2002, S. 176ff. 
Angehörigen niederreißen. Es mag nicht auszuschließen sein, dass von derlei Maßnahmen präventive Wirkung ausgehen kann, doch erscheint selbst dies fraglich: So können staatlich veranlasste Häuserzerstörungen in den Augen von Terroristen das augenfälligste Beispiel von „Staatsterrorismus“ darstellen und hierdurch sogar eine subjektive Legitimation für ihr Tun liefern. Vor allem jedoch gilt in einem Rechtsstaat das Gebot, dass der legitime Zweck der Sicherheit nicht jedes Mittel heiligt, sondern nur ein solches, das sich mit den freiheitssichernden und die Menschenwürde achtenden Standards der Verfassung verträgt. ${ }^{40}$ Kollektivsanktionen sind deswegen problematisch, da sie Personen treffen können, die zur Tat keinen hinreichenden Bezug haben. Indem Sanktionen gegen die Familie eines Selbstmordattentäters verhängt werden, um ein bei diesem intaktes Wertebewusstsein ansprechen zu können, werden die nicht involvierten Familienangehörigen zu Objekten staatlicher Maßnahmen degradiert, was einen Verstoß gegen die Menschenwürde darstellt. ${ }^{41}$ Wurde weiter oben ausgeführt, dass die moderne Staatlichkeit nicht den unbedingten Vorrang einer einzelnen Meinung - einer einzelnen materialen Wahrheit - kennt, so ist dies an dieser Stelle dahingehend zu präzisieren, dass der moderne Staat - der Rechtsstaat - auch nicht den unbedingten Vorrang der Sicherheit kennt. Der moderne Staat darf in der Wahrnehmung seiner Schutzfunktion vor dem Terrorismus nicht seinerseits der terroristischen „Logik des Absoluten “42 anheim fallen. Wäre dies anders, so wäre er totalitär.

Doch ist es letztlich auch gar nicht nötig, gegenüber Terroristen nach Alternativen zur hergebrachten strafrechtlichen Präventionswirkung zu suchen, denn in einer gewissen Weise wirkt sie auch hier. Zwar spricht gegen die Möglichkeit einer Integration von Terroristen mittels strafrechtlichen Diskurses, dass ein funktionierender Diskurs herrschaftsfrei verlaufen muss, ${ }^{43} \mathrm{um}$ vernünftige Ergebnisse produzieren zu können. Spätestens seit dem Deutschen Herbst ${ }^{44}$ ist aber klar, dass die Bundesrepublik mit Terroristen nicht (mehr) verhandelt. Die staatliche Friedensordnung ist nicht relativierbar und damit auch nicht verhandelbar. Ein politischer Dialog mit Terroristen ist eine Stufe, die erst dann erklommen werden kann, wenn diese das staatliche Gewaltmonopol anerkannt haben - dann freilich wären es auch keine Terroristen mehr. ${ }^{45}$ Jedoch stehen diese Überlegungen nicht der präventiven Anwendung des Strafrechts gegenüber Terroristen entgegen. Das Strafrecht verhandelt nicht es droht an, verspricht, sanktioniert oder erlässt. Die Debatte über die strafrechtliche Präventionswirkung darf dabei nicht immer nur von völlig verblendeten Extremtätern ausgehen. Ebenso wie es unter „gewöhnlichen“ Straftätern nicht nur solche gibt, auf die in keiner Weise präventiv eingewirkt werden kann, gibt es auch in Terrorgruppen Zweifler und Mitläufer, die

40 Josef Isensee, a.a.O., S. 83, 106

41 Vgl. Bernd Grzeszick, a.a.O., S. 55, 71f.

42 Bernd Grzeszick, a.a.O., S. 55, 71.

43 Vgl. Jürgen Habermas, Faktizität und Geltung, Frankfurt a. M.1998, S. 18ff., $152 \mathrm{ff}$.

44 Als Deutschen Herbst bezeichnet man die Monate September und Oktober 1977. Am 5. September 1977 entführte die $R A F$ den damaligen Arbeitgeberpräsidenten Hanns Martin Schleyer. Ziel der Aktion war es, inhaftierte RAFMitglieder freizupressen. Um den Druck auf die deutsche Bundesregierung zu erhöhen, wurde zudem am 13. Oktober 1977 durch eine Gruppe palästinensischer Terroristen ein mit Passagieren besetztes deutsches Flugzeug entführt. Die Bundesregierung ging jedoch nicht auf die Forderung der Entführer ein, die inhaftierten $R A F$-Mitglieder freizulassen.

45 Vgl. Bernd Grzeszick, a.a.O., S. 55, 70. es von Seiten der Rechtsordnung zurückzugewinnen gilt. Darüber hinaus verlangt zumindest auch die Theorie der positiven Generalprävention eine Bestrafung desjenigen Terroristen, der von der Richtigkeit seines Tuns völlig überzeugt ist. $\mathrm{Zu}$ bedenken sind überdies auch die Konsequenzen, die drohen würden, wenn sich ein Strafrecht vollkommen von der Idee der Präventionswirkung verabschieden würde. Gefahrenabwehr würde dann über das jetzige Maß hinaus von Geheimdiensten in die Hand genommen, da kaum davon auszugehen ist, dass staatliche Interventionen in diesem Bereich unterbleiben würden. Die schützende Funktion des Strafverfahrensrechts ginge hier völlig verloren. ${ }^{46}$

Strafrechtliche Prävention gegenüber Terroristen kann außerdem auch in der Weise funktionieren, dass terroristische Vorbereitungshandlungen unter Strafe gestellt werden. Über die von einer gesetzlichen Strafandrohung ausgehende generalpräventive Wirkung hinaus kann hierdurch auch die Verhinderung konkret drohender Terrorakte gewährleistet werden. Ein Beispiel für eine derartige Kriminalisierung von Vorbereitungshandlungen stellt die Organisationsstraftat der Bildung terroristischer Vereinigungen nach § 129a des Strafgesetzbuchs (StGB) dar. Entscheidend für das Vorliegen einer terroristischen Vereinigung im Sinne dieser Strafbestimmung ist die Verfolgung eines Vereinigungszwecks, der in der Begehung ${ }^{47}$ oder Androhung ${ }^{48}$ bestimmter Straftaten ${ }^{49}$ besteht. Im Verhältnis zur Begehung der terroristischen Katalogtaten stellen sämtliche der in § 129a StGB genannten Tatbestandsmodalitäten - die Gründung und Mitgliedschaft, ${ }^{50}$ die Unterstützung ${ }^{51}$ sowie die Werbung $^{52}$ - in materieller Hinsicht Vorbereitungshandlungen dar. Auch durch die zeitliche Vorverlagerung der Strafbarkeit zieht das Strafrecht mithin Prävention an sich. ${ }^{53}$

Wenn oben die These in Erwägung gezogen wurde, dass der Terrorist für die deutsche Strafrechtsordnung ein ungewohntes Subjekt ist, so will der Autor dies nicht in einem Sinne verstanden wissen, dass die deutsche Strafrechtsordnung den Terroristen als ihren Feind betrachtet, denn dies käme einer Vorenthaltung der Idee der positiven Spezialprävention gegenüber Terroristen gleich. Die Bestimmung des $§ 46$ Abs. 1 S. 2 StGB gebietet im Rahmen der Strafzumessung jedoch gerade die Berücksichtigung der Wirkungen, die von der Strafe für das künf-

46 Bettina Weißer JZ 2008, S. 388, 394f.

$47 \S 129$ a Abs. 1, Abs. 2 StGB.

48 § 129a Abs. 3 i.V.m. § 129a Abs. 1, Abs. 2 StGB.

49 § 129a Abs. 1 Nr. 1 StGB nennt Mord (\$ 211 StGB) und Totschlag (§ 212 StGB) sowie Taten nach $\S \S 6$ bis 12 Völkerstrafgesetzbuch. $\S 129$ a Abs. 1 Nr. 2 StGB verweist auf Straftaten gegen die persönliche Freiheit in den Fällen des § 239a oder $§ 239 b$ StGB.

$\S 129$ a Abs. 2 StGB zählt als Katalogtaten zum Beispiel die schwere Körperverletzung nach $\S 226$ StGB oder die Brandstiftung nach $\S 306$ StGB auf. Da diese Taten im Vergleich zu den in $\S 129$ a Abs. 1 StGB aufgelisteten grundsätzlich einen geringeren Unrechtsgehalt aufweisen, verlangt § 129a Abs. 2 StGB a.E. als zusätzliches Tatbestandsmerkmal, dass die angestrebte Tat „bestimmt ist, die Bevölkerung auf erhebliche Weise einzuschüchtern, eine Behörde oder eine internationale Organisation rechtswidrig mit Gewalt oder durch Drohung mit Gewalt zu nötigen oder die politischen, verfassungsrechtlichen, wirtschaftlichen oder sozialen Grundstrukturen eines Staates oder einer internationalen Organisation zu beseitigen oder erheblich zu beeinträchtigen, und durch die Art ihrer Begehung oder ihre Auswirkungen einen Staat oder eine internationale Organisation erheblich schädigen kann“ (vgl. hierzu: Bettina Weißer JZ 2008, S. 388, 389).

$50 \S 129$ a Abs. 1, Abs. 2, Abs. 3 StGB.

51 § 129a Abs. 5 S. 1 i.V.m. § 129a Abs. 1, Abs. 2, Abs. 3 StGB.

$52 \S 129$ a Abs. 5 S. 2 i.V.m. § 129a Abs. 1, Abs. 2 StGB.

53 Bettina Weißer JZ 2008, S. 388, 394; vgl. hierzu: Bernd Heinrich, Die Grenzen des Strafrechts bei der Gefahrprävention, in: ZStW 1/2009, S. 112, $115 \mathrm{ff}$. 
tige Leben des Täters in der Gesellschaft zu erwarten sind. Zwar lassen sich Terroristen, die die Idee der modernen Staatlichkeit verneinen, unter Umständen als Feinde dieser Staatlichkeit betrachten, doch sollte man sich eines sehr vorsichtigen Umgangs mit dem Begriff des Feindes bewusst sein. Jedenfalls gilt die Kategorie des Feindes im Recht nur in sehr beschränktem Maße: So erlaubt das Kriegsrecht unter Umständen die bewusste Schädigung eines feindlichen Staates, ${ }^{54}$ das Strafrecht kennt demgegenüber weder Freund noch Feind. ${ }^{55}$ Es ist nicht die Aufgabe des Strafrechts, eine Feindeserklärung von Extremisten anzunehmen. ${ }^{56}$ Ein Strafrecht, dessen Ziel es wäre, Verbrecher als Feinde zu vernichten, wäre von vornherein nicht in der Lage, mit ihnen zu kommunizieren. ${ }^{57} \mathrm{Ob}$ strafrechtliche Kommunikation - in Form von Prävention - vom (potenziellen) Straftäter angenommen wird, ist eine andere Frage. Sie darf jedoch nicht von vornherein vorenthalten werden.

Geht man damit von der Grundannahme aus, dass das Strafrecht auch im Verhältnis zu Terroristen Präventionswirkungen zu entfalten vermag, so stellt sich zugleich die Frage nach den Grenzen. Das „Paradigma der Zweckmäßigkeit“58 würde für sich genommen eine grenzenlose Bekämpfung des Terrorismus verlangen. Prävention ist ebenso wie Gefahrenabwehr prinzipiell schrankenlos, da die ihr dienenden Eingriffe so lange gerechtfertigt sind, wie die Gefahr andauert. ${ }^{59}$ Schon oben, im Rahmen der Zurückweisung von Kollektivsanktionen, wurde aber bereits darauf hingewiesen, dass der Rechtsstaat im Rahmen der Bewältigung des Phänomens des Terrorismus nicht völlig grenzenlos agieren darf. Auch wenn der Rechtsstaat den Terroristen unter Umständen nicht verstehen mag, muss er ihm gegenüber gleichwohl nach Normen handeln, ${ }^{60}$ hat er Grenzen einzuhalten. Kein Streit dürfte darüber bestehen, dass sich diese Grenzen aus dem Völkerrecht, den Menschenrechten, den rechtsstaatlichen Prinzipien sowie dem Grundgesetz ergeben. Wenn hinsichtlich der Frage, dass dies so sein muss, Konsens besteht, ist damit aber noch nicht schlüssig dargetan, warum dies so ist. Die bloße Normgeltung mag als Antwort nicht zu befriedigen, da diese mitunter nur ein aktueller Zustand sein kann, der - zumindest jenseits der Grenzen von Art. 79 Abs. 3 des Grundgesetzes - nahezu beliebig geändert werden kann, wenn denn die politischen Mehrheitsverhältnisse dies zulassen. Die Antwort dürfte wiederum in der Institution der modernen Staatlichkeit zu finden sein, die es als Errungenschaft zu bewahren gilt. Zwar ist richtig, dass dem Rechtsstaat eine Schutzfunktion gegenüber seinen Bürgern zukommt, ${ }^{61}$ jedoch impliziert dies keinen absoluten Vorrang der Sicherheit. Im Gegenteil: Es gibt keine für jeden und jederzeit zu gewährleistende Sicherheit. Sicherheit ist ein paradoxer Begriff: Die Gewährleistung absoluter körperlicher Sicherheit für eine bestimmte Person könnte theoretisch nur durch sanktionierbare Einschränkungen gegenüber anderen Personen und demnach durch den

54 Gerd Roellecke JZ 2006, S. 265, 266.

55 Vgl. demgegenüber den von Günther Jakobs geprägten Begriff des Feindstrafrechts (Günther Jakobs Bürgerstrafrecht und Feindstrafrecht, in: HRRS 3/2004, S. 88ff. [passim])

56 Vgl. Thomas Groß, Terrorbekämpfung und Grundrechte, in: KJ 2002, S. 1, 6.

57 Vgl. Cornelius Prittwitz, a.a.O., S. 499, 514.

58 Bettina Weißer JZ 2008, S. 388, 394.

59 Winfried Hassemer WestEnd. Neue Zeitschrift für Sozialforschung 2/2006, S. $75,79$.

60 Gerd Roellecke JZ 2006, S. 265, 269

61 Vgl. Gerd Roellecke JZ 2006, S. 265, 268.
Verlust an Sicherheitsempfindung erreicht werden. ${ }^{62}$ Würde ein Staat alle ihm möglichen Mittel einsetzen, um beispielsweise Leib und Leben seiner Bürger zu schützen, so könnte dieses Ziel nur in einem gut funktionierenden Polizeistaat erreicht werden. Folge wäre eine ständig gegebene latente subjektive Unsicherheit, Adressat einer nicht vorhersehbaren staatlichen Maßnahme zu werden. ${ }^{63}$ Günter Frankenberg spricht insoweit von „struktureller Angst“ potenzieller Normadressaten, die aufkommen kann, wenn Normen im Hinblick auf die ihre Anwendung auslösenden Tatbestandsmerkmale zu unbestimmt bleiben. ${ }^{64}$ Das Postulat des Friedens verbietet aber gerade die totalitäre Durchsetzung von Sicherheit. Würde der Staat gegen dieses Postulat verstoßen, würde er sich durch sein totales Streben nach Sicherheit in seinem Absolutheitsanspruch auf eine Stufe mit Terroristen stellen. ${ }^{65}$ Weiter noch: Er würde sich selbst aufgeben. Häufig ist dies gerade das Kalkül von Terroristen, um ihre subjektive moralische Legitimation verstärken zu können. Der Rechtsstaat tut jedoch gut daran, auf dieses Kalkül nicht hereinzufallen. ${ }^{66}$

Eine weitere Grenze, die der Rechtsstaat im Umgang mit dem Terrorismus zu wahren hat, wird im Strafrecht durch das Schuldprinzip gezogen, das in seiner Ausprägung als Gewährleistung eines angemessenen Verhältnisses zwischen Schuld und Sanktion gegenüber der präventiven Wirkung des Strafrechts limitierende Funktion hat. ${ }^{67}$ Die Bedeutung dieses Prinzips gilt es gerade in Zeiten zu betonen, in denen sich das moderne Strafrecht vermehrt zu einem Gefahrenabwehrrecht entwickelt - eine Entwicklung, die momentan unter dem Eindruck der öffentlichen Angst vor Terroranschlägen zu beobachten ist. ${ }^{68}$ Als Beispiele hierfür seien nur die zunehmende Vertypung von Gefährdungen als strafrechtliches Unrecht sowie die Debatte ${ }^{69}$ um ein Feindstrafrecht zu nennen. ${ }^{70}$

Wenngleich das Paradigma der Prävention dem Grunde nach nicht in Frage gestellt werden soll, so darf dessen Berücksichtigung gleichwohl nicht zu einer Überdehnung strafrechtlicher Sanktionen führen. Auf den ersten Blick bemerkenswert ist insoweit die bereits erwähnte Vorschrift des § 129a StGB. Diese Bestimmung enthält im Vergleich zu den in ihr enthaltenen Katalogtaten nicht nur eine zeitliche Vorverlagerung der Strafbarkeit, sondern teilweise auch erheblich höhere Strafrahmen. Beispielsweise sieht das Delikt der Zerstörung wichtiger Arbeitsmittel gemäß § 305a Abs. 1 StGB als Sanktion eine Freiheitsstrafe bis zu fünf Jahren oder eine Geldstrafe vor. Gründet jemand eine terroristische Vereinigung, deren Zweck einzig und allein auf die Zerstörung wichtiger Arbeitsmittel gerichtet ist - § 305a StGB stellt eine Katalogtat nach $§ 129$ a Abs. 2 Nr. 2 StGB dar -,

62 Vgl. Günter Frankenberg, Nochmals: Angst im Rechtsstaat, in: WestEnd. Neue Zeitschrift für Sozialforschung 2/2006, S. 55, 61.

63 Vgl. Klaus Günther, Angst im Rechtsstaat, in: WestEnd. Neue Zeitschrift für Sozialforschung 2/2006, S. 49, 52.

64 Günter Frankenberg, WestEnd. Neue Zeitschrift für Sozialforschung 2/2006, S. 55, 56.

65 Vgl. Bernd Grzeszick, a.a.O., S. 55, 80.

66 Vgl. Hans-Heiner Kühne, a.a.O., S. 103, 108

67 Vgl. Peter-Alexis Albrecht, Die vergessene Freiheit, 2. Aufl., Berlin 2006 S. $69 \mathrm{ff}$.

68 Winfried Hassemer, WestEnd. Neue Zeitschrift für Sozialforschung 2/2006, S. $75,81$.

69 Vgl. hierzu: Günther Jakobs HRRS 3/2004, S. 88ff. (passim); Hans-Heiner Kühne, a.a.O., S. 103, 105; Cornelius Prittwitz, a.a.O., S. 499, 505.

70 Vgl. Winfried Hassemer, WestEnd. Neue Zeitschrift für Sozialforschung 2/2006, S. 75,81 . 
so droht das Gesetz in § 129a Abs. 2 i.V.m. Abs. 1 StGB eine deutlich höhere Strafe an, nämlich eine Freiheitsstrafe von einem Jahr bis zu zehn Jahren, ohne dass zur Begehung der Katalogtat auch nur unmittelbar angesetzt worden sein muss. ${ }^{71}$ Die Problematik dieser Strafrahmenanhebung hält sich freilich dann in Grenzen, wenn man berücksichtigt, dass § 129a Abs. 2 StGB a.E. zusätzlich zur angestrebten Begehung der Katalogtat verlangt, dass diese „bestimmt ist, die Bevölkerung auf erhebliche Weise einzuschüchtern, eine Behörde oder eine internationale Organisation rechtswidrig mit Gewalt oder durch Drohung mit Gewalt zu nötigen oder die politischen, verfassungsrechtlichen, wirtschaftlichen oder sozialen Grundstrukturen eines Staates oder einer internationalen Organisation zu beseitigen oder erheblich zu beeinträchtigen, und durch die Art ihrer Begehung oder ihre Auswirkungen einen Staat oder eine internationale Organisation erheblich schädigen kann".

\section{Schlussbetrachtung}

Zweifellos stellt das Bemühen um die innere Sicherheit den Rechtsstaat im Kampf gegen den Terrorismus vor neue Herausforderungen. Was allerdings das Strafrecht anbelangt, hat

71 Kritisch hierzu: Bettina Weißer JZ 2008, S. 388, 391. die Untersuchung ergeben, dass dieses Rechtsgebiet auch das Phänomen des Terrorismus zu erfassen vermag. Der Terrorist unterscheidet sich zwar durch seine inneren Überzeugungen grundlegend von einem „gewöhnlichen“ Kriminellen, jedoch kann strafrechtliche Prävention auch gegenüber jenem wirken. Auch in Terrorgruppen gibt es Zweifler und Mitläufer, die es von Seiten der Rechtsordnung zurückzugewinnen gilt. Darüber hinaus verlangt zumindest auch die Theorie der positiven Generalprävention eine Bestrafung desjenigen Terroristen, der von der Richtigkeit seines Tuns völlig überzeugt ist.

Selbstverständlich hat der Rechtsstaat im strafrechtlichen Kampf gegen Terrorismus Grenzen zu wahren. Eine absolute Sicherheit kann ohnehin niemals gewährleistet werden. Sicherheit ist ein paradoxer Begriff, da staatliche Handlungen zum Schutz bestimmter Rechtsgüter regelmäßig zu Eingriffen in andere führen. Hinzu käme ein Verlust an Sicherheitsempfindung, wenn der Staat mit vergleichsweise unbestimmten Straftatbeständen auf terroristische Aktivitäten reagieren würde. Würde der Rechtsstaat die in dieser Abhandlung aufgezeigten Grenzen im Kampf gegen den Terrorismus überschreiten, würde er sich nicht nur selber aufgeben, sondern es hieße zugleich, dem Terrorismus durch ein Abdriften des Staates in den Totalitarismus einen weiteren Sieg zu schenken.

\section{Perspektiven einer demokratischen Sicherheit}

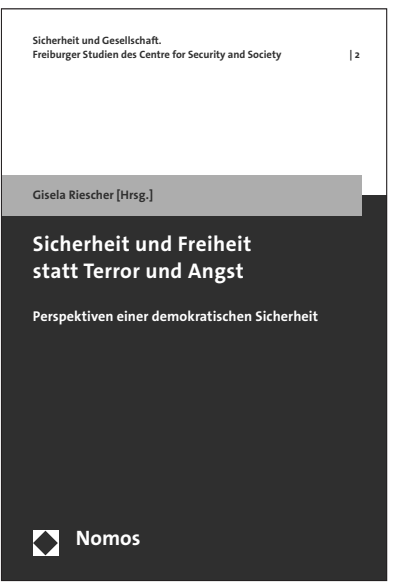

Sicherheit und Freiheit statt Terror und Angst Perspektiven einer demokratischen Sicherheit Herausgegeben von Gisela Riescher

2010, 298 S., brosch., 39,- $€$, ISBN 978-3-8329-5496-3

(Sicherheit und Gesellschaft. Freiburger Studien des Centre for Security and Society, Bd. 2)

Die demokratische Lebensform auch in Zeiten des Terrors zu erhalten, ist mehr denn je Aufgabe demokratischer Politik, sie zu diskutieren, ist Aufgabe der Wissenschaften. Politikwissenschaftliche, juristische und philosophische Beiträge bilden die kontrovers geführten Debatten um Sicherheit und Freiheit, Terror und Angst ab und führen sie kritisch weiter.

Bitte bestellen Sie im Buchhandel oder versandkostenfrei unter $\downarrow$ www.nomos-shop.de

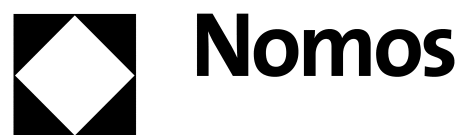

